\title{
Bone fracture risk: density and microarchitecture qualification
}

\author{
F. Cosmi ${ }^{\mathrm{a}}$, A. Nicolosi ${ }^{\mathrm{b}}$, G. Saviola ${ }^{\mathrm{c}}$ \\ ${ }^{a}$ Università di Trieste, DIA, via A. Valerio 10, 34127 Trieste, Italy, E-mail: cosmi@ units.it \\ ${ }^{b}$ M2TEST srl, Padriciano 99, Trieste, Italy,E-mail: alessandra@bestest.it \\ 'IRCCS Maugeri Clinical Scientific Institutes, Pavia, Italy, E-mail: gianantonio.saviola@icsmaugeri.it
}

\begin{abstract}
Osteoporosis is a systemic skeletal disease where an increase in bone fragility is due to low bone mass and micro-architectural deterioration of bone tissue, which occur over a long period of time without clinical significance.

Currently, Double Energy X-ray Absorption (DEXA) is the gold standard for bone mineral density assessment and the diagnosis of osteoporosis, but the majority of fractures occur in patients who would not be considered at fracture risk based on their Bone Mineral Density (BMD) values. It has long been known that fracture risk depends not only on mass loss, but also on bone architecture, whose alterations are an independent factor of increased fragility.

The Bone Elastic Structure Test, BES TEST ${ }^{\circledR}$, is a recently introduced analysis that assesses bone quality as expressed by the elastic properties of the trabecular micro-architecture from a virtual biopsy of the patient and could be a helpful add-on to densitometry for predicting fragility fractures and patient monitoring.

The aim of this study is the comparison of DEXA and BES TEST ${ }^{\circledR}$ ability as 3-year risk estimators in a clinical application. In the CONTROL group, the BSI T-score was significantly different from the femoral DEXA T-score ( $\mathrm{p}=0.0005$.In the FRACTURED group, the BSI T-score is significantly different from both the femoral DEXA T-score $(\mathrm{p}=0.0266)$ and the lumbar DEXA T-score $(\mathrm{p}=0.0051)$. Inter-group t-test statistical analysis (95\% significance) shows that the femoral DEXA T-score (neck) of the CONTROL and the FRACTURED groups are not significantly different ( $\mathrm{p}=0.1478)$, while the BSI T-score of the CONTROL and the FRACTURED groups highlights a significant difference $(\mathrm{p}=0.0001)$. Despite the small number of subjects, our data seem to confirm that the BSI could be a helpful add-on to densitometry for predicting fragility fractures and patient monitoring.
\end{abstract}

Keywords: BES TEST; Bone Elastic Structure Test; Trabecular structure; Bone quality; DEXA; Osteoporosis

\section{Introduction}

Osteoporosis is a systemic skeletal disease where an increase in bone fragility is due to low bone mass and microarchitectural deterioration of bone tissue [1], which occur over a long period of time without clinical significance. With an increase in lifetime expectancy, osteoporosis (OP) and bone fragility have become a major healthcare burden on the patient, the family and society, causing decreased autonomy, a not negligible mortality rate and important healthcare costs, already exceeding $€ 37$ billion/year in Europe [2].

Currently, Double Energy X-ray Absorption (DEXA) is the gold standard for bone mineral density assessment and the diagnosis of osteoporosis. The results of densitometry examinations are expressed as a T-score, that is a number representing a person's bone density compared with the average bone density value of young Caucasian women, who are at peak bone density. A DEXA T-score $>-1$ is considered normal, a $-2.5 \leq$ DEXA T-score $\leq-1$ is classified as osteopenia (diminished bone density) and a DEXA T-score $<-2.5$ corresponds to osteoporosis. It should be noted, though, that over half of the fragility fractures in the population arise in women that would not be considered at risk based on Bone Mineral Density (BMD) alone [3,4]. Characteristics like bone geometry, size and mineralization degree, 
hydroxyapatite crystal size and heterogeneity, collagen properties, osteocyte density, trabecular and cortical microarchitecture are all features influencing the ability of a bone to withstand trauma. Because of these independent factors, the individual risk of fracture may indeed rise or drop, even without meaningful changes in BMD [5,6]. For this reason, fracture risk assessment tools like FRAX and DEFRA [7], where developed, mainly to validate treatment prescriptions. Bone turnover markers, indices of bone remodeling, are also useful for monitoring the patient by assessing the response to therapy and treatment adherence [8].

Bone tissue includes:

- compact (or cortical) bone, smooth and hard, is located on the bone and shaft surface, conferring the necessary rigidity;

- trabecular (or cancellous) bone, found in the two epiphyses (extremities), constitutes a complex lightweight optimized structure, somehow similar to a sponge or a system of bars and in healthy conditions, conferring elasticity and resilience;

- bone marrow: it fills the inner cavity of cortical and cancellous bone.

The cortical BMD accounts for $80 \%$ of our skeleton, but bone mechanical resistance strongly depends also on the internal spatial arrangement of bone structure, that has long been considered the second key factor of bone load-bearing capacity, in addition to bone density, Fig. 1 . Although cancellous bone accounts for only $20 \%$ of our skeleton, the trabecular structure is an additional key factor responsible for bone resistance, and the majority of fractures occur in patients who would not be considered at fracture risk based on their BMD values, leading to the hypothesis that DEXA might not represent the elective exam, or be completely reliable, since fracture risk depends not only on mass loss, but also on bone architecture, whose alterations are an independent factor of increased fragility.

The Bone Elastic Structure Test, BES TEST ${ }^{\circledR}$, is a recently introduced analysis that assesses bone quality as expressed by the elastic properties of the trabecular micro-architecture and can measure its alterations induced by age, pathological conditions or lack of exposure to physiological mechanical stimuli.

The aim of this study is the comparison of DEXA and BES TEST ${ }^{\circledR}$ ability as 3-year risk estimators in a FRACTURED and in a CONTROL group of women of similar age.

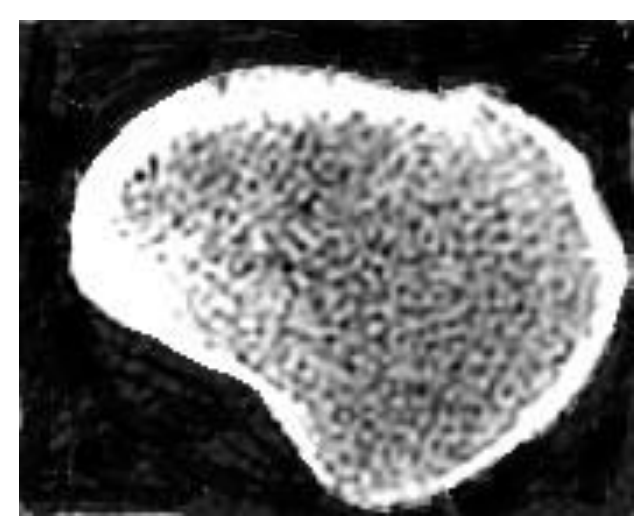

Fig. 1. CT scan femoral neck section: outer compact part and internal cancellous structure.

\section{Materials and methods}

\subsection{Bone quality Cell Method model}

The BES TEST is based on an application of the Cell Method (CM), a recently introduced discrete method $[9,10]$. The CM theory is deeply different from that of other widely used numerical methods such as FEM and brings some advantages with it. Since CM is a discrete method, field equations are directly written for a whole region and no differentiation is needed to form the solving equations. Thus, CM can be employed in all those cases in which variables cannot be differentiated, for example when the displacement field undergoes large variations. Even when the size of the heterogeneities is the same scale of that of the mesh, a CM model can be solved with consistent results. 
For a brief description of the Cell Method for plane elasticity, let us consider a body of constant thickness $t$, loaded in a plane. The variables used in the problem can be classified in:

- configuration variables, that is kinematic variables such as nodal coordinates and displacements, strain tensor, etc.;

- source variables, that is static and dynamic variables such as forces, torques, momenta, etc.;

- energy variables, which always result from the product of a configuration by a source variable, and which we are not going to use in the following.

From this classification, the method derives the use of two staggered meshes: configuration variables are associated with the $n$ nodes of a primal complex of cells, while source variables are associated with a dual complex, the only condition being that each node of the primal cell be surrounded by a dual cell, or influence region for the inner node. An example is depicted in Fig.2.
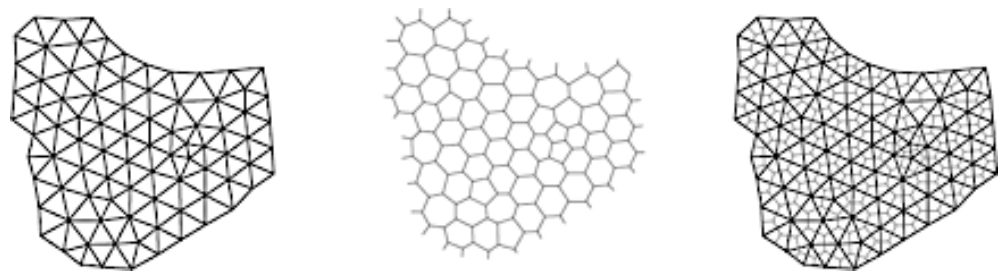

Fig. 2. Primal (left) and dual (center) complexes of cells with the resulting staggered mesh (right).

Once the interpolation displacement field over the primal cell and the constitutive matrix for the primal cell $[D]_{c}$ are defined, the constitutive equation for each cell can be expressed in the usual form

$$
\{\sigma\}_{\mathrm{c}}=[\mathrm{D}]_{\mathrm{c}}\{\varepsilon\}_{\mathrm{c}}=[\mathrm{D}]_{\mathrm{c}}[\mathrm{B}]_{\mathrm{c}}\{u\}_{\mathrm{c}}
$$

where

- $\{\sigma\}_{\mathrm{c}}$ collects the stress components;

- $[D]_{c}$ represents Hooke law for the isotropic homogeneous material of the primal cell;

- $\{\varepsilon\}_{c}$ collects the strain components:

- $\{u\}_{\mathrm{c}}$ collects the nodes displacements.

With reference to Fig.3, equilibrium condition for the influence region $\tilde{U}_{h}$ of the node $h$ is obtained by adding up all the forces acting on the dual cell:

$$
\boldsymbol{T}_{h}+\boldsymbol{F}_{h}+\boldsymbol{B}_{h}=0
$$

where

- $\boldsymbol{T}_{h}$ total force acting on the boundary of $\tilde{U}_{h}$, due to all the cells that surround node $h$;

- $\boldsymbol{B}_{h}$ resultant external force acting on $\tilde{U}_{h}$ through the boundary cells,;

- $\boldsymbol{F}_{h}$ resultant of volume forces and of external forces acting on $\tilde{U}_{h}$ through the boundary cells.

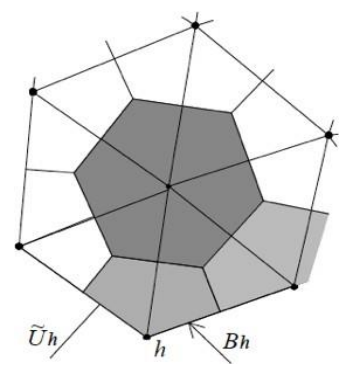

Fig. 3. Equilibrium of the dual cell of node $h$. 
Equation (2) represents a set of $2 n$ linear equations in the $2 n$ unknowns $u_{i x}, u_{i y}(i=1, \ldots, n)$ which can be also written as

$$
[\mathrm{K}]\{u\}=\{F\}
$$

and solved with the usual methods.

A detailed description of CM is beyond the purpose of this paper, but it should be mentioned that some aspects of the Cell Method may recall the Finite Volumes Method or the so-called direct - or physical - approach [11]. All these methods did not have much success, maybe due to an intrinsic difficulty in developing higher order elements. On the contrary, higher order interpolation functions are easily implemented in CM. Generally, CM results agree with those obtained with other numerical methods, although convergence and accuracy are better than those obtained with FEM with the same interpolation order. Overall, the $\mathrm{CM}$ approach appears to be particularly interesting from the point of view of computation time, memory requirements and accuracy of the results [12,13,14].

In the BES TEST ${ }^{\circledR}$ application of CM, a radiographic virtual biopsy of the patient, acquired in the proximal phalanges of the non-dominant hand, is converted into a structural model and the elastic response to compressive loads is computed, Fig.4, [15 - 18].

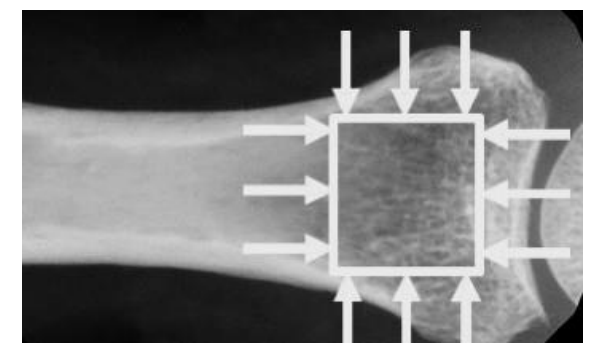

Fig. 4. Virtual biopsy of the patient and simulations.

The apparent elastic moduli, $E^{*}$, are then combined in an index that gives an indication of the quality of the bone structure, the BSI (Bone Structure Index):

$$
B S I=\mathrm{a}_{1}\left(\mathrm{~b}_{1} E^{*}-\mathrm{b}_{2} C F\right)
$$

where $\mathrm{a} 1$ is a value function of the acquisition parameters, $\mathrm{b} 1$ and $\mathrm{b} 2$ are positive constants and $C F$ is linked to the mineral content in the examined region.

Analogously to DEXA, bone structure quality as measured by the $B S I$ can also be interpreted using the T-score concept. Following the usual clinical interpretation, the BES TEST ${ }^{\circledR}$ results expressed in terms of BSI_T-score compare the patient's $B S I$ with the mean $B S I$ value for young Caucasian women (age 20-45) and measure this difference as the number of standard deviations (SD), as illustrated in Tab.1.

Table 1. Clinical reading of BSI.

\begin{tabular}{ll}
\hline Bone Structure Quality & $B S I$ T-score \\
\hline Normal & $\geqslant-1$ \\
First level alteration & $<-1$ or $\geqslant 2.5$ \\
Significant alteration & $<2.5$ \\
\hline
\end{tabular}

This study compares the DEXA and BES TEST ${ }^{\circledR}$ ability as 3-year risk estimators in a clinical application.

\subsection{Study population}

The examined FRACTURED population consists of 12 Caucasian women with a recent atraumatic fragility, fracture, notwithstanding a normal or osteopoenic DEXA scan result both at the femoral neck and at the lower spine (lumbar). Age in this group was 39 - 74 years, with a Mean (SD) value of 62.4 (10.8) years. 
The CONTROL population consists of 15 Caucasian women who had not suffered any fragility fractures before a bone quality evaluation by BES TEST ${ }^{\circledR}$ in 2015, performed within the study described in detail in [19], and who confirmed their state of health by interview, stating that they had not suffered any fragility fracture in the following three years. The BSI T-score and the DEXA femoral neck T-score were available for all the examined subjects, whose age ranged $47-74$ years, with a Mean (SD) value of 64.7 (8.4) years.

DEXA and BES TEST ${ }^{\circledR}$ analyses were performed by different operators, blind to any previous evaluation.

\section{Results}

Usefulness of BES TEST ${ }^{\circledR}$ as an add-on to DEXA is clearly shown in the diagram below, Fig.5, where different conditions can be recognized for the patients studied in this work.

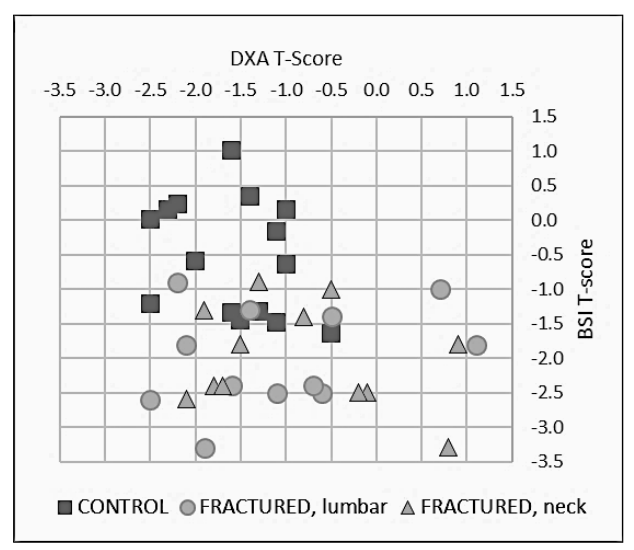

Fig. 5. Quality vs quantity: the BSI T-score vs. the DEXA T-score.

It can be noted that:

- all CONTROL patients fall in the top left quadrant: a low DEXA T-score and high BES TEST ${ }^{\circledR} \mathrm{T}$-score indicate low mineral content but high resistance capability of the bone structure;

- all FRACTURED patients lie in the bottom half of the diagram: the DEXA T-score spans results that indicate a condition of high or slightly compromised mineral content, but the low BES TEST ${ }^{\circledR}$ T-score is significant of a compromised resistance capability of the bone structure.

There is no correlation between the BSI and the DEXA T-scores, as shown in in Tab.2, regardless of the densitometry assessment site. This result is to be expected, as quality and quantity are independent factors of bone resistance, and confirms the findings of previous works [19-21].

Table 2. Correlation between DEXA T-score and BSI T-score.

\begin{tabular}{lll}
\hline Population & DEXA Site & $\mathrm{R}^{2}$ \\
\hline CONTROL & Femoral (neck) & 0.086 \\
FRACTURED & Lumbar (lower spine) & 0.057 \\
FRACTURED & Femoral (neck) & 0.083 \\
\hline
\end{tabular}

This is further confirmed by intra-group t-test (Student's test [22]) statistical analysis (95\% significance):

- In the CONTROL group, the BSI T-score is significantly different from the femoral DEXA T-score ( $\mathrm{p}=0.0005)$;

- In the FRACTURED group, the BSI T-score is significantly different from both the femoral DEXA T-score $(\mathrm{p}=0.0266)$ and the lumbar DEXA T-score $(\mathrm{p}=0.0051)$.

The DEXA and BES TEST ${ }^{\circledR}$ results are summarized in Table 3 and depicted in Fig. 6. 
Table 3. DEXA and BES TEST ${ }^{\circledast}$ results: Mean (SD)

\begin{tabular}{lll}
\hline Population & CONTROL & FRACTURED \\
\hline BSI T-score & $-0.5(0.8)$ & $-2.0(0.7)$ \\
Femoral DEXA (neck) & $-1.6(0.6)$ & $-1.1(1.1)$ \\
Lumbar DEXA (lower spine) & -- & $-0.9(1.0)$ \\
\hline
\end{tabular}

Inter-group t-test statistical analysis (95\% significance) shows that:

- the femoral DEXA T-score (neck) of the CONTROL and the FRACTURED groups are not significantly different $(\mathrm{p}=0.1478)$;

- the BSI T-score of the CONTROL and the FRACTURED groups highlights a significant difference $(\mathrm{p}=0.0001)$.
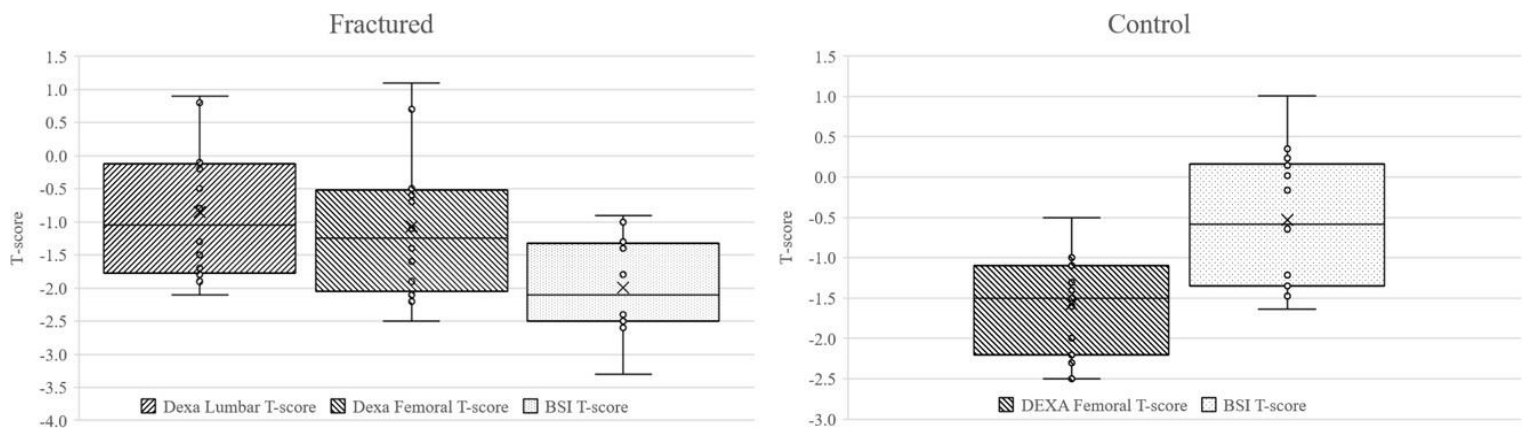

Fig. 6. Box diagrams (min, max, mean, SD) for the FRACTURED (left) and the CONTROL (right) groups.

\section{Discussion and conclusions}

As average human lifespan is growing, a parallel rise in the rate of osteoporosis is projected to pose an increasing burden on society in future years. As observed in several studies, about half of those at risk of fracture still go undetected. Since it is widely accepted that the mechanical properties of bone depend on both its composition and its trabecular component micro-architectural arrangement, the fracture risk in these patients appears to be linked to alterations of the trabecular architecture. The combination of both densitometry and quality assessment is likely to improve diagnostic accuracy and new methods are needed to assess the trabecular structure elastic properties.

Despite the small number of subjects, our data seem to confirm that the BSI could be a helpful add-on to densitometry for predicting fragility fractures and patient monitoring.

While a major fracture hardly goes unnoticed, defining a heathy control population in osteoporosis is particularly difficult because, as discussed above, a correct diagnosis can be obtained only in a fraction of cases and a major fracture may, and often does, appear without any warning symptom.

In this work, the difficulty of a correct diagnosis of the risk of fracture has partially been solved by referring to the retrospective 3 years risk evaluation.

The quality vs. quantity diagram may offer further help in clinical practice, since the quadrant in which each patient's data are located is representative of the patient's conditions and could therefore drive both the prognosis and the choice of the appropriate treatment.

The $B S I$ assessment requires a very low X-ray dose in a peripheric site, making the BES TEST ${ }^{\circledR}$ a very safe test that can be performed, if necessary, even at a young age. Instead of the 18-24 months interval required by DEXA to detect significative fluctuations, the relatively quick response of the trabecular structure to changes implies that modifications can be detected by BES TEST ${ }^{\circledR}$ after several weeks. The treating physician could thus monitor the bone response to any therapeutic strategy and tailor the patient's treatment every few months if necessary, providing patients with a strong motivation to strictly adhere to their therapy and avoid discontinuation.

Further work is needed to confirm these findings in larger groups. 


\section{Acknowledgements}

This work would not have been possible without the cooperation of the patients. The Authors are grateful to S. Sacco, L. Saibene, and L. Abdi-Ali for their contribution in the development of this work.

\section{References}

[1] Lane JM et al. Osteoporosis. Clin Orthop Relat Res, 372 (2000) 139-150.

[2] Kanis JA et al. Osteoporos. December (2013) 8:144.

[3] Pasco JA et al. Osteoporos Int 17 (2006) 1404-9.

[4] Schuit SCE et al. Bone 34 (2004) 195-202.

[5] Farkhondeh P et al. Ther. Clin. Risk. Manag. 14 (2018) 2029-2049.

[6] Fonseca $\mathrm{H}$ et al. Sports Med. 44 (2014) 37-53.

[7] Kanis JA et al. Osteoporos Int. 22 (2011) 2395-411.

[8] Rossini M et al. Calcif Tissue Int. 91 (2012) 365-9

[9] Tonti E. CMES 1 (2001) 11-26

[10] Tonti E. J Comp Physics, 257 (2014) 1260-1290

[11] Huebner, K.H. (1995) The finite element method for Engineers, John Wiley \& Sons, 39-59

[12] Cosmi, F. CMES 2 (2001) 1-7

[13] Cosmi, F. CMES 8 (2005) 191-200

[14] Cosmi, F. CMES 74 (2011) 269-282

[15] Cosmi, F., Mazzoleni G. 29 (2014) 517- 528

[16] Cosmi, F. MCB12 (2015) 87-105

[17] Cosmi F, Tomanik M. Materials Today: Proceedings 3 ( 2016 ) 947 - 952

[18] Cosmi F, Maximova N. Materials Today: Proceedings 12 (2019) 246-251

[19] Cosmi F et al. Materials Today: Proceedings, 5 (2018) 26772-26777

[20] Saviola G. et al. Minerva Medica, June 2019

[21] Cosmi F, Saracchini S. Materials Today: Proceedings, 7 (2019) 455-462

[22] Kalpić D., Hlupić N., Lovrić M. (2011) Student’s t-Tests. In: Lovric M. (eds) International Encyclopedia of Statistical Science. Springer, Berlin, Heidelberg, 